\title{
Hybrid Pixel EELS Detector: Low Noise, High Speed, and Large Dynamic Range
}

Benjamin Plotkin-Swing ${ }^{1}$, Tracy Lovejoy ${ }^{1}$, Niklas Dellby ${ }^{1}$, George Corbin ${ }^{1}$, Matthew Hoffman ${ }^{1}$, Sacha De Carlo ${ }^{2}$, Luca Piazza ${ }^{2}$, Chris Meyer ${ }^{1}$, Andreas Mittelberger ${ }^{1}$ and Ondrej Krivanek ${ }^{1}$

${ }^{1}$ Nion Co., Kirkland, Washington, United States, ${ }^{2}$ Dectris, Baden-Daettwil, Aargau, Switzerland

Direct electron detectors have been very successful in transmission electron microscopy [1], with applications including Cryo-EM [2] and STEM diffraction pattern detection [3,4]. The detectors can be separated into two broad classes: back-thinned CMOS detectors with small pixels that are optimized for energies of 200-400 keV and work best in low-dose regimes used in biological EM [1,2], and hybrid-pixel detectors using thicker detection layers and large pixels, which have fewer pixels but can handle larger incident beam currents, and are at their optimum at 20-200 keV [3,4]. Here we demonstrate the suitability of a hybrid-pixel detector for electron energy loss spectroscopy (EELS).

The detector, Dectris ELA mounted on a Nion IRIS EELS as part of Nion HERMES microscope, has a large dynamic range, narrow point spread function, $\mathrm{DQE}(0) \sim 0.8$ even without single electron arrival discrimination, and it is resilient to radiation damage. It can detect up to $0.8 \mathrm{pA}$ per pixel and hence a $>100$ pA EELS zero-loss peak (ZLP) without saturation, if the ZLP is spread over $>125$ pixels. At the same time, it can reliably detect isolated single electrons in the high loss region of the spectrum. There are 1030x 514 pixels of 75 um each. Fig. 1a shows an EEL spectrum of boron nitride with $10^{7}$ dynamic range in which an unsaturated zero loss peak is read out at the same time as inner shell loss edges, with $105 \mathrm{pA}$ total beam current. The ZLP is spread over 130 pixels in the non-dispersion direction. The relationship between current and the raw detector counts becomes non-linear above $0.1 \mathrm{pA} / \mathrm{pixel}$, but a ZLP count accurate to better than $1 \%$ is automatically recovered with count-rate correction, shown in orange.

Figure 1 also shows that the detector characteristics in terms of DQE and MTF with 30-200 keV primary electrons are near to ideal [5], especially the MTF for primary electrons $60 \mathrm{keV}$ and below (fig. 1b). At $200 \mathrm{kV}$, the DQE at zero spatial frequency is 0.9 (fig. 1c). The detector uses a selectable threshold to exclude low energy events, and this results in essentially zero instrumental dark current and read noisethe noise limit for low intensity signals typically comes from the cosmic ray background intrinsic to working on the surface of the earth. Its maximum frame rate at 16-bit digital resolution is 2250 full frames per second.

In fig. 2 these characteristics are all put to practical use to produce a very high SNR spectrum image (SI) of a semiconductor interface acquired as a series of 32 fast SIs with $128 \times 128$ scan pixels and 0.5 ms per spectrum (about 8s per image). The series of SIs are sub-pixel aligned based on concurrently acquired HAADF images. Unlike traditional detectors where read noise introduces a high cost for summing many low-intensity spectra, the noise in the summed SI spectra is equivalent to a single SI with 32 times longer pixel dwell time. Breaking the acquisition into fast frames allows per-frame drift correction, and the integration can be cut off after-the-fact when the beam damage threshold has been exceeded. This technique can also reduce damage to dose-rate sensitive samples. The high dynamic range allows the ZLP to be acquired in the same spectrum, which is useful for analysis (quantification, deconvolution, etc..) and yields a low-noise simultaneous image similar to bright-field. 

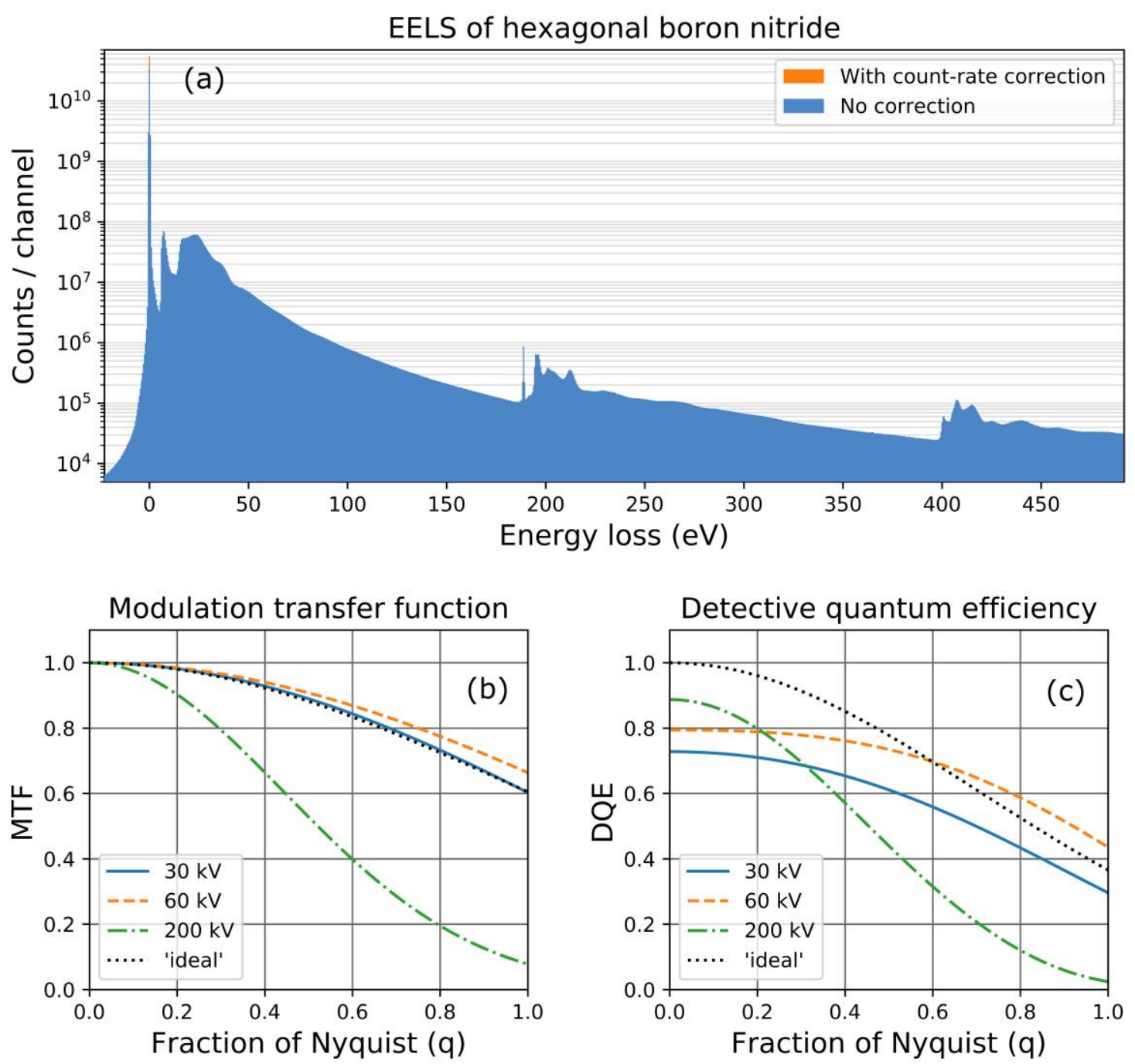

Figure 1. (a) EELS of hexagonal boron nitride with $\mathrm{E} 0=60 \mathrm{keV}$, beam current $=105 \mathrm{pA}, 100$ s exposure, $\alpha / 2=27 \mathrm{mrad}, \beta / 2=50 \mathrm{mrad}$. and $0.5 \mathrm{eV} / \mathrm{channel}$. This is raw data with no dark subtraction or gain normalization. (b) The modulation transfer function and (c) the detective quantum efficiency of the ELA direct detector at 30,60, and $200 \mathrm{kV}$ microscope operating voltage.
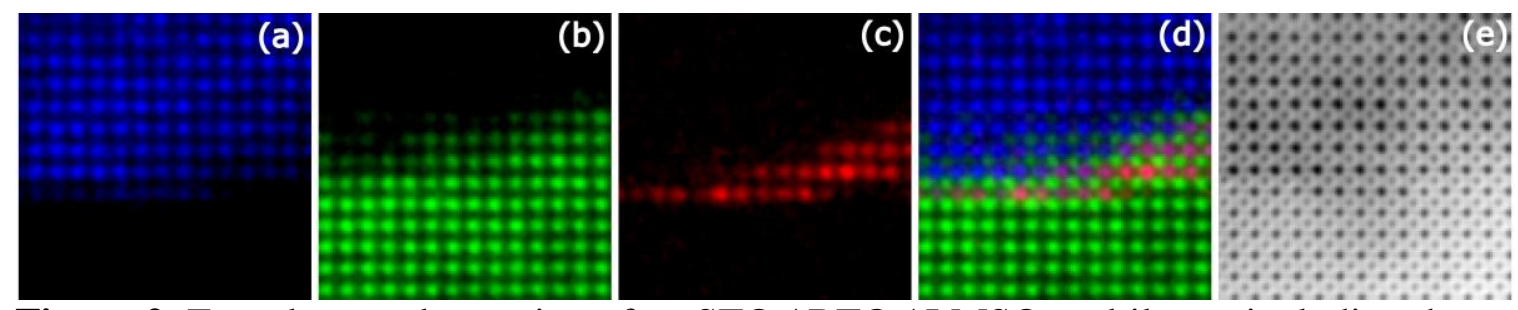

Figure 2. Fast elemental mapping of an STO / BTO / LMSO multilayer, including the unsaturated ZLP, acquired as 32 separate $128 \times 128$ SIs of 8 s each, aligned and summed (4.3 min total). (a) map of La M4,5 edge. (b) map of Ti L2,3 edge. (c) map of Ba M4,5 edge. (d) RGB composite map. (e) ZLP intensity map, similar to a bright-field. 


\section{References}

[1] A.R. Faruqi et al., Nucl. Instruments Methods Phys. Res. Sect. A 878 (2018) 180-190.

[2] K. Naydenova et al., IUCrJ. 6 (2019) 1086.

[3] M.W. Tate et al., Microsc. Microanal. 22 (2016) 237.

[4] J.A. Mir et al., Ultramicroscopy 182 (2017) 44.

[5] B. Plotkin-Swing et al. in preparation (2020). 